\title{
Enantiomeric selectivity in behavioural and electrophysiological responses of Aedes aegypti and Culex quinquefasciatus mosquitoes
}

\author{
J.I. Cook ${ }^{1}$, S. Majeed ${ }^{2}$, R. Ignell ${ }^{2}$, J.A. Pickett ${ }^{1}$, M.A. Birkett ${ }^{1}$ \\ and J.G. Logan ${ }^{3 *}$ \\ ${ }^{1}$ Centre for Sustainable Pest and Disease Management, Chemical Ecology \\ Group, Biological Chemistry Department, Rothamsted Research, \\ Harpenden, Hertfordshire, AL5 2JQ, UK: ${ }^{2}$ Division of Chemical Ecology, \\ Department of Plant Protection Biology, PO Box 102, Sundsvägen 14, \\ SLU 230 53, Alnarp, Sweden: ${ }^{3}$ Department of Disease Control, Faculty of \\ Infectious and Tropical Diseases, London School of Hygiene \& Tropical \\ Medicine, Keppel Street, London, WC1E 7HT, UK
}

\begin{abstract}
1-Octen-3-ol is a kairomone for many haematophagous insects including mosquitoes. Numerous studies have examined the effects of racemic 1-octen-3-ol; however, few studies have investigated the role of individual enantiomers in relation to mosquito attraction. In the present study, we investigated the behavioural and electrophysiological responses of two mosquito species, Aedes aegypti and Culex quinquefasciatus, to individual enantiomers and mixtures of 1-octen-3-ol, employing a laboratory Y-tube olfactometer and single sensillum recordings. The olfactory receptor neurons of both Ae. aegypti and $C x$. quinquefasciatus had a significantly higher response to the $(R)$-1-octen-3-ol enantiomer compared to the (S)-1-octen-3-ol

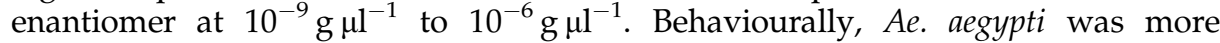
responsive to the $(R)-1$-octen-3-ol enantiomer, showing an increase in flight activity and relative attraction compared to $C x$. quinquefasciatus. The (R)-1-octen-3-ol enantiomer caused an increase in activation for $C x$. quinquefasciatus. However, the most notable effect was from an (R:S)-1-octen-3-ol mixture (84:16) that caused significantly more mosquitoes to sustain their flight and reach the capture chambers (demonstrated by a reduced non-sustained flight activity), suggesting that it may have a behaviourally excitatory effect. For $C x$. quinquefasciatus, a reduced relative attraction response was also observed for all treatments containing the (R)-1-octen-3-ol enantiomer, either on its own or as part of a mixture, but not with the (S)-1-octen-3-ol enantiomer. This is the first time enantiomeric selectivity has been shown for Ae. aegypti using electrophysiology in vivo. The implications of these results for exploitation in mosquito traps are discussed.
\end{abstract}

Keywords: mosquito, 1-octen-3-ol, enantiomer, electrophysiology, behaviour

(Accepted 22 February 2011)

\footnotetext{
*Author for correspondence

Fax: + 44 (0)207 9272918

E-mail: james.logan@lshtm.ac.uk
} 


\section{Introduction}

Haematophagous insects, including mosquitoes (Diptera: Culicidea), use vision, heat and humidity, but predominantly volatile chemical cues, known as kairomones, to locate potential vertebrate hosts (Khan et al., 1966; Eiras \& Jepson, 1994; Gibson \& Torr, 1999). One of the most extensively studied kairomones for haematophagous insects is 1-octen-3-ol. This compound, which exists in two enantiomeric forms, is released by many vertebrates and plant species (Hall et al., 1984; Knudsen et al., 1993; Kline et al., 2007). 1-Octen-3-ol was first described in relation to haematophagous insects as a component of ox odour and has since been shown to be effective at increasing catches of tsetse flies (Diptera: Glossinidae) (Bursell, 1984; Hall et al., 1984; Vale \& Hall, 1985a,b). In combination with carbon dioxide $\left(\mathrm{CO}_{2}\right)$, a major component of vertebrate breath, 1-octen-3-ol has also been shown to increase behavioural responses and trap catches of mosquitoes, including those of the genera Aedes and Anopheles (Vythilingam et al., 1992; Kemme et al., 1993; Becker et al., 1995; Kline \& Lemire, 1995; Mboera et al., 2000; Burkett et al., 2001; Russell, 2004; Miller et al, 2005; Kline et al., 2007). Interestingly, 1-octen-3-ol was found to have little behavioural effect on most Culex spp. mosquitoes, despite the fact that some have olfactory receptors on their antennae and maxillary palps (Takken \& Kline, 1989; Hill et al., 2009).

1-Octen-3-ol is found in cattle breath to be predominantly (R)-1-octen-3-ol, varying between 80:20 R:S and 92:8 R:S (Hall et al., 1984). Despite this, most investigations on haematophagous insects have assessed this compound only in its racemic form $(1: 1, R: S)$. Furthermore, the racemic form of 1-octen-3-ol is used in commercially available insect traps, such as the Mosquito Magnet and Midgeaters (Vythilingam et al., 1992; Kemme et al., 1993; Becker et al., 1995; Mboera et al., 2000; Burkett et al., 2001; Russell, 2004; Miller et al., 2005). Although no differences in either electroantennogram (EAG) activity, behaviour in a wind tunnel or attractiveness in the field between the $(R)$ and the $(S)$ enantiomers have been found for tsetse flies (Bursell, 1984; Hall et al., 1984; Vale \& Hall, $1985 a, b)$, there is evidence that mosquitoes can discriminate between the enantiomers. For example, a study by Kline et al. (2007) showed an increase in numbers of Anopheles crucians and Ochlerotatus infirmatus caught in traps when using the (R)-1-octen-3-ol enantiomer compared to the (S)-1-octen-3-ol enantiomer. In line with this, a recent study showed that an olfactory receptor neuron (ORN) in the maxillary palps of $C x$. quinquefasciatus mosquitoes displayed 'remarkable sensitivity' to the (R)-1-octen-3-ol enantiomer compared to the (S)-1-octen-3-ol enantiomer (Syed \& Leal, 2007). Similarly, an odorant receptor of the yellow fever mosquito, Ae. aegypti, has been shown to be enantioselective in vitro, responding predominantly to the (R)-1-octen-3-ol enantiomer, when heterologously expressed in Xenopus oocytes (Bohbot \& Dickens, 2009). Therefore, one might expect to see stronger, as yet undefined, behavioural responses of Aedes and Culex species mosquitoes to the $(R)$ enantiomer. However, this has not been investigated to date and is further complicated by the fact the Ae. aegypti and Cx. quinquefasciatus have different host preferences. Aedes aegypti is a known anthropophilic mosquito that responds preferentially to human host odours, as well as other mammals, and Cx. quinquefasciatus preferentially feeds on birds (Takken \& Kline, 1989; Takken, 1991; Zinser et al., 2004). 1-Octen-3-ol has been identified from human odour previously; therefore, Ae. aegypti would be expected to show positive behavioural responses to the compound. To our knowledge, 1-octen-3-ol has not been identified from bird odour, and so its ecological role is difficult to predict for $C x$. quinquefasciatus mosquitoes.

The aim of this study was to further our understanding on the role of 1-octen-3-ol and to provide possible explains for the inconsistency in trapping efficacy of previous studies. To achieve this, we investigate the behavioural and electrophysiological effect of pure enantiomers of 1-octen-3-ol and mixtures on the attraction of Ae. aegypti and Cx. quinquefasciatus mosquitoes using laboratory based olfactometer bioassays and electrophysiology employing single-sensillum recordings (SSRs).

\section{Materials and methods}

Behaviour

\section{Insects}

Aedes aegypti mosquitoes, refm strain, were originally obtained from the London School of Hygiene and Tropical Medicine and were kept in culture in the laboratory at Rothamsted Research. The culture was maintained in a controlled environment at $27 \pm 2^{\circ} \mathrm{C}, 55-60 \%$ relative humidity (RH) and a L:D 12:12 h photoperiod. The larvae were fed daily with Tetramin ${ }^{\circledR}$ Tropical Fish Flakes according to larval density of rearing trays. Adult insects were kept in BugDorm-1 (MegaView, Taiwan) plastic rearing cages and provided with $10 \%$ sucrose solution (Fischer Scientific UK Ltd, UK) on cotton wool. Females were blood-fed weekly on sheep blood in Alsevers (TCS Biosciences, UK) using a Hemotek ${ }^{\circledR}$ Membrane Feeding System (Discovery Workshops, UK).

Culex quinquefasciatus mosquitoes were originally obtained from the Thai strain provided by the Liverpool School of Tropical Medicine. Similar to Ae. aegypti, this culture was maintained at Rothamsted Research in a controlled environment at $27 \pm 2^{\circ} \mathrm{C}, 55-60 \% \mathrm{RH}$, although the photoperiod was inverted, D:L 12:12 h. Larvae were fed regularly with Brewer's Yeast Tablets (500 mg) (Holland \& Barrett, UK). Adults were kept in BugDorm-1 rearing cages and were fed on defibrinated horse blood (TCS Biosciences, UK) weekly.

For behavioural bioassays, female mosquitoes aged between 5-12 days that had not been previously blood-fed, were collected and stored in plastic pre-release chambers (WHO, Malaysia), without sucrose and water, in the bioassay room for two hours, allowing time for the insects to acclimatize.

\section{Behavioural assays}

A Y-tube olfactometer (Logan et al., 2008), comprising a Y-shaped Plexiglass tube (id $62 \mathrm{~mm}$; fig. 1B-D) that allows insects to move towards odours coming from the upwind end, was used for the behavioural bioassays. Air was pumped through an activated charcoal filter then a glass bell jar (51), containing distilled water $\left(\mathrm{dH}_{2} \mathrm{O}, 1 \mathrm{l}\right.$; fig. 1G-I) prior to being split between the two arms of the olfactometer. Flow meters regulated the airflow in each arm at $101 \mathrm{~min}^{-1}$ (fig. 1F). Chemicals in solvent (hexane) were introduced onto filter paper $(10 \mu \mathrm{l}$; Whatman No. 1, 9-cm diameter filter papers, Whatman plc, UK) by micropipette (Drummond, UK) and placed in sintered glass expander/reductor couplings (fig. 1E). A second pump provided a charcoal filtered airflow over the chemical samples, regulated at $20 \mathrm{ml} \mathrm{min}^{-1}$, and this was introduced through polytetrafluoroethylene (PTFE) tubing 


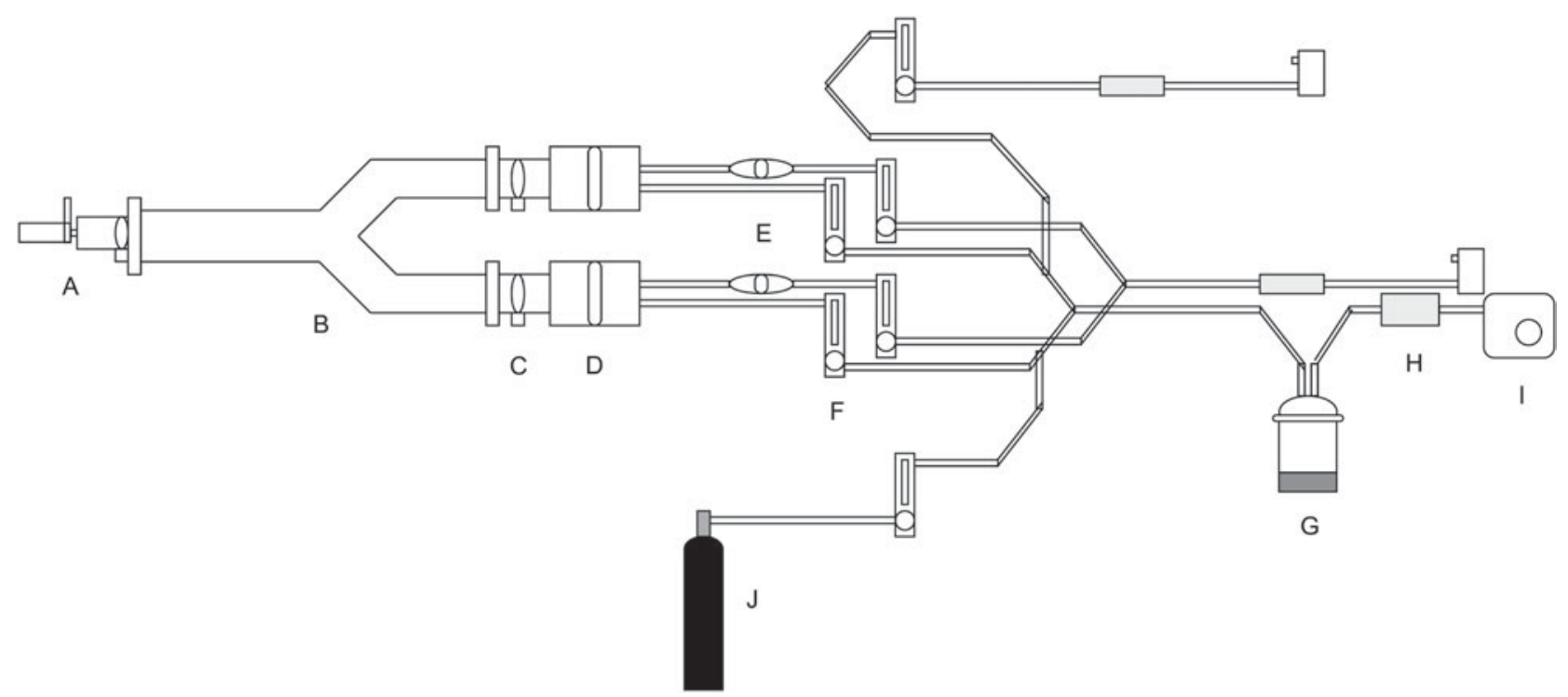

Fig. 1. Y-tube olfactometer bioassay design. Female insects were collected in pre-release chambers (A) and allowed to acclimatize for two hours. These chambers were designed to easily attach to the main Y-tube olfactometer (B). Air was pumped (I) through a charcoal filter (H) and humidified in a glass bell jar containing $\mathrm{dH}_{2} \mathrm{O}(\mathrm{G})$. The airflow was regulated by flowmeters (F). Chemicals were applied to filter papers and placed in sintered glass vessels $(\mathrm{E})$. Where necessary, $\mathrm{CO}_{2}$ was introduced from a cylinder located in the bioassay room (J).

adjacent to the main airflow. For dose response experiments, chemicals were introduced in the presence of $\mathrm{CO}_{2}$ presented via a pressurized cylinder (BOC, UK) and introduced directly into the main airflow before the split, or in the required tubing arm after the split (fig. 1J). Airflow was regulated by a flow meter $\left(20 \mathrm{ml} \mathrm{min}^{-1}\right)$ and was balanced in the opposite arm of the Y-tube by the addition of another pump introducing charcoal filtered air at the same flow rate (fig. 1). The Y-tube olfactometer and components were changed after every third treatment and cleaned thoroughly using distilled water and Tepol (Harvey, Waddington, UK) then allowed to air dry. Additionally, the PTFE tubing was rinsed with ethanol, and the sintered glass vessels were cleaned with acetone and distilled water. Both the tubing and vessels were then placed for two hours at $180^{\circ} \mathrm{C}$ in an oven.

In all experiments, three behavioural parameters were recorded: relative attraction (the proportion of mosquitoes in the arm of the Y-tube containing the odour stimulus out of the total number of mosquitoes in both arms); upwind flight activity (the proportion of mosquitoes that had flown upwind beyond $30 \mathrm{~cm}$ in the stem of the Y-tube) and non-sustained flight activity (NSFA; the proportion of mosquitoes that had flown beyond $30 \mathrm{~cm}$ in the stem of the Y-tube, but did not reach the capture chambers at the end of the arms).

\section{Dose response experiments}

Racemic 1-octen-3-ol, (R)-1-octen-3-ol, (S)-1-octen-3-ol and an $(R: S)$-1-octen-3-ol mixture (84:16) were prepared in hexane and serial diluted ten-fold to provide solutions of $10^{-12}-10^{-4} \mathrm{~g} \mu \mathrm{l}^{-1}$. Aliquots of $10 \mu \mathrm{l}$ were applied in each replicate, giving doses of $10^{-11}-10^{-3} \mathrm{~g} \mathrm{\mu l}^{-1}$ (treatment vs. blank). All treatments were tested with $\mathrm{CO}_{2}$. In addition to the nine treatment doses, three controls were also included: (i) $\mathrm{CO}_{2}$ vs. blank, (ii) $\mathrm{CO}_{2}$ vs. $\mathrm{CO}_{2}$ (to ensure there was no bias in the Y-tube set up) and (iii) aqueous L-(+)-lactic acid $+\mathrm{CO}_{2}$ vs. distilled water $\left(\mathrm{dH}_{2} \mathrm{O}\right)$ (a positive control). Experiments were done using a semi-randomized block design. Treatments were randomized then ordered by ascending dose within the groups that occurred between equipment changes/washes. Twelve replicates were conducted and the randomisation also included orientation of the Y-tube olfactometer, so six replicates were conducted with the test compound in the left arm and six in the right arm.

A pre-release chamber containing approximately 20 female mosquitoes was attached to the release chamber and the door opened to allow the mosquitoes to move. The cover of the visual occlusion frame was replaced, the main airflow turned on and the insects were given five minutes to acclimatize. During this time, the treatments were prepared on filter papers as above, allowing one minute for the solvent to evaporate. After acclimatization, the papers were introduced into the sintered glass vessels, and the secondary airflows were turned on along with the $\mathrm{CO}_{2}$ and the balancing airflow. Once all flow meters had settled, the insects were released for a duration of $90 \mathrm{~s}$. The swivel doors on the capture chambers and release chambers were closed, and the position of all insects in the stem and arms was recorded. The cover to the visual occlusion frame was removed, and the insects in the capture chambers and release chambers were counted. The $\mathrm{CO}_{2}$ cylinder, as well as the balancing airflow, was turned off. The insects in the olfactometer were removed by suction. Experiments were conducted in the light with Ae. aegypti and under red-light (artificial night) with Cx. quinquefasciatus.

\section{Electrophysiology}

\section{Insects}

Aedes aegypti, Rockefeller strain, and Culex quinquefasciatus, Thai strain (obtained as eggs from Rothamsted Research Institute, UK), were used for electrophysiology experiments. 
Both mosquito species were cultured at SLU, Alnarp. The cultures were maintained at $27^{\circ} \mathrm{C}$, with a relative humidity of $70 \%$ at an L:D 12:12 h photoperiod (the photoperiod was inverted for $C x$. quinquefasciatus, D:L 12:12 h). Adults were maintained in mesh and plastic culture cages with free access to $10 \%$ sucrose through a filter paper wick. Larvae were reared in a separate chamber $\left(27^{\circ} \mathrm{C}, 60 \% \mathrm{RH}\right.$ and $\left.12: 12 \mathrm{~L}: \mathrm{D}\right)$ in distilled water-filled plastic trays $(20 \mathrm{~cm} \times 30 \mathrm{~cm} \times 10 \mathrm{~cm})$. Larvae were kept in groups of $\sim 500$ per tray with free access to fish food (Tetramin ${ }^{\circledR}$ Tropical Fish Flakes). Pupae were collected daily in $30 \mathrm{ml}$ containers and transferred to the adult cages. For electrophysiology, non-blood-fed female mosquitoes were chosen at random from a population aged between three and five days.

\section{Insect preparation}

To obtain a stable recording, a female mosquito was anesthetized $\left(1.0-1.5 \mathrm{~min}\right.$ at $\left.-20^{\circ} \mathrm{C}\right)$ and mounted over double-sided tape on a microscope slide $(76 \times 26 \mathrm{~mm})$. To secure the insect, half of the thorax and the entire abdomen were covered by tape fixed to the microscope slide. The cover slip, partially covered with double-sided tape, was positioned abutting the mosquito head, above and at a right angle to the proboscis. Maxillary palps were lifted and extended smoothly onto the cover slip. The scales that cover the basiconic sensilla on the maxillary palps were removed by gentle rubbing. The mounted mosquito was placed in the single sensillum recording (SSR) rig under a Nikon Eclipse (E600FN) microscope. The maxillary palps were visualized at high magnification $(750 \times)$.

\section{Single sensillum recording}

Maxillary palps of mosquitoes house three types of olfactory receptor neurons, and their extracellular spikes can easily be resolved into three distinct populations based on differences in amplitude. In order to determine a comparative olfactory response profile for the olfactory receptor neurons to the three forms of 1-octen-3-ol, SSRs were performed on ten maxillary palp basiconic sensilla from each culicine species, Ae. aegypti and $C x$. quinquefasciatus.

Two tungsten electrodes were electrolytically sharpened ( $\sim 1-\mu \mathrm{m}$ tip diameter) by dipping repeatedly in a $10 \% \mathrm{KNO}_{2}$ at $5-10 \mathrm{~V}$. The reference electrode was inserted into the eye of the mosquito under low magnification $(75 \times)$. The recording electrode was connected to a preamplifier $(10 \times$, Syntech, Kirchzarten, Germany) and inserted into the base or shaft of a basiconic sensillum to extracellularly record the activity of the olfactory receptor neurons housed within the sensilla. The preamplifier was connected to an analogue to digital signal converter (IDAC, Syntech), which in turn was connected to a PC computer for data acquisition and storage. The data were analyzed with Autospike v.3.0 (Syntech).

\section{Stimuli and stimulus delivery}

Seven concentrations of racemic 1-octen-3-ol, $(R)$-1-octen3-ol and (S)-1-octen-3-ol enantiomers were used to describe the dose-response relationships. Each of the three volatiles was dissolved in hexane to make a stock solution of $10 \mu \mathrm{g} \mu \mathrm{l}^{-1}$. Aliquots of $10 \mu \mathrm{l}$ of each serially diluted compound

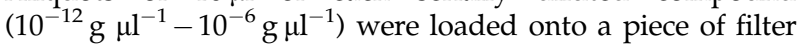
paper $(5 \times 10 \mathrm{~mm})$ and placed into the glass Pasteur pipette.
Hexane $(10 \mu \mathrm{l})$ alone was used as a control. All pipettes were prepared in a fume hood and left for $40 \mathrm{~min}$ to ensure the evaporation of the hexane solvent.

A continuous humidified air stream delivered at $1.51 \mathrm{~min}^{-1}$ through a glass tube $(10 \mathrm{~mm}$ id) passed over the maxillary palp preparation. Stimuli were applied through a hole in the glass tube, which was $11 \mathrm{~cm}$ away from the outlet. A stimulus controller (Syntech, Germany) was used to pass $0.51 \mathrm{~min}^{-1}$ through the stimulus cartridge for $500 \mathrm{~ms}$ into the continuous air stream through the hole in the glass tube. Fresh stimulus cartridges were applied for each new replicate. Throughout this study, synthetic $\mathrm{CO}_{2}$-free air (Scan Gas, Sweden) was used for the main airflow and stimulus delivery. One-to-two recordings were taken from each mounted insect.

\section{Chemicals}

Racemic 1-octen-3-ol was obtained from Alfa-Aeser (Heysham, Lancashire, UK). (R)-1-Octen-3-ol and (S)-1octen-3-ol were provided by Botanix Ltd (Paddock Wood, Kent, UK), and Agrisense BCS Ltd (Pontypridd, Gwent, UK). The enantiomeric purity/composition of materials was determined by derivitization to respective butyrate esters using pyridine and butyric anhydride. The esters were then analysed by high resolution gas chromatography (GC), using an Agilent 6890 GC equipped with a $\beta$-cyclodextrin column ( $25 \mathrm{~m} \times 0.32 \mathrm{~mm}$ id $\times 0.25 \mu \mathrm{m}$ film thickness), cool on-column injector and a flame ionization detector (FID). The following oven temperature programme was used: $40^{\circ} \mathrm{C}$ per $1 \mathrm{~min}$, then $3^{\circ} \mathrm{C} \mathrm{min}{ }^{-1}$ to $150^{\circ} \mathrm{C}$, hold for $0.1 \mathrm{~min}$, then $5^{\circ} \mathrm{C} \mathrm{min}^{-1}$ to $180^{\circ} \mathrm{C}$, hold for $15 \mathrm{~min}$. The derivatized enantiomers resolved clearly and their composition was determined by analysis of the resultant peak areas. Enantiomeric purity was determined to be: $(R)-(-)-1$-octen-3-ol, 99\%; (S)-(+)-1-octen-3-ol, 99\%; $(R: S)-1-o c t e n-3-o l, 84: 16$ and racemic 1-octen-3-ol (50:50).

\section{Statistical analysis}

For behavioural data, a generalized linear model (GLM) with binomial distribution and logistic regression was used to perform pairwise comparisons between the $\mathrm{CO}_{2}$ or blank control and the test treatments (Genstat ${ }^{\circledR}, 12$ th edn). The mean proportions of mosquitoes responding to each treatment were compared. Initially, the model blocked by day and layered out each of the controls to determine where effects were occurring. Following this, the model was simplified to only block by day to compare all treatments. Predictions, taking into account total variance, were produced along with standard errors and Least Significant Differences (LSDs). Results are shown with significance levels $(P<0.05, P<0.01, P<0.001)$.

The electrophysiological data were analyzed using a twoway repeated measures ANOVA followed by Bonferroni post test (Graph Pad Prism vs. 5.0a).

\section{Results \\ Behaviour}

\section{Relative attraction}

To determine the optimum concentration and composition of 1-octen-3-ol enantiomers to elicit a behavioural response, behavioural bioassays were performed in the laboratory. In all cases, with Ae. aegypti, the positive control (L-(+)-lactic 
(a) Racemic 1-octen-3-ol

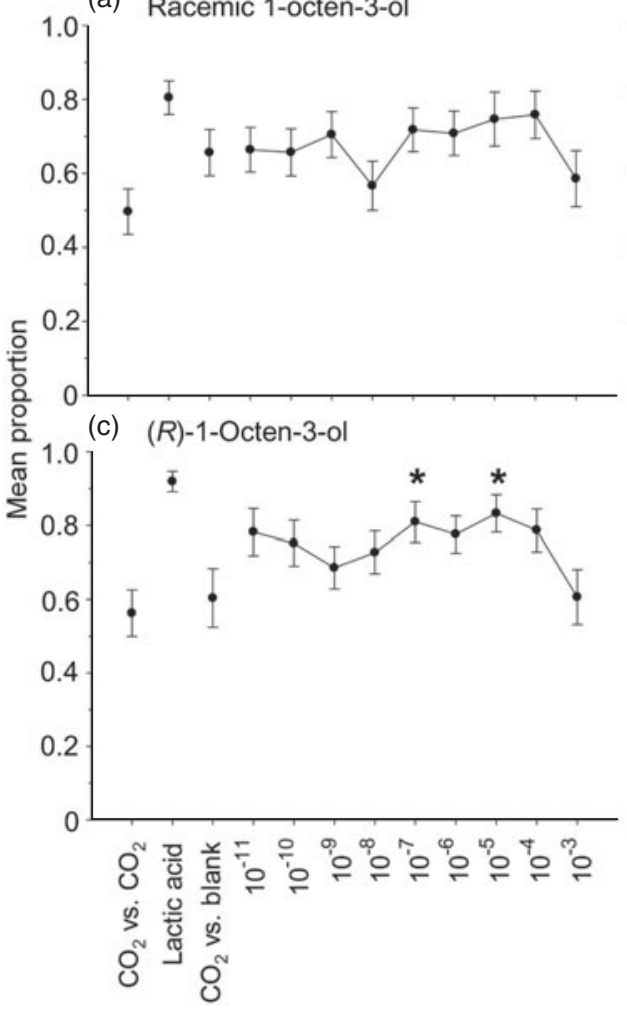

(b)

(S)-1-Octen-3-ol

$\Phi$

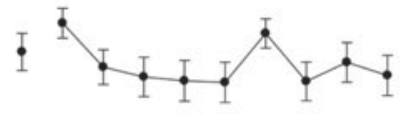

I

(d) (R:S)-1-Octen-3-ol (84:16)

Ð

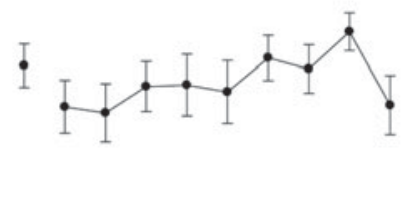

Treatment/dose (g)

Fig. 2. Relative attraction of Aedes aegypti female mosquitoes in a Y-tube olfactometer to 1-octen-3-ol treatments (plus $\mathrm{CO}_{2}$ ) and controls: $\mathrm{CO}_{2}$ vs. blank, $\mathrm{CO}_{2}$ vs. $\mathrm{CO}_{2}$ and $\mathrm{L}-(+)$-lactic acid $+\mathrm{CO}_{2}$ vs. $\mathrm{dH}_{2} \mathrm{O}$. Relative attraction is the proportion of mosquitoes that flew upwind and were caught in the capture chamber of the treated arm of the Y-tube olfactometer. (a) racemic 1-octen-3-ol, (b) (S)-1-octen-3-ol, (c) (R)-1-octen-3-ol and (d) (R:S)-1-octen-3-ol (84:16) mixture. Asterisks show statistically significant differences in response compared to the $\mathrm{CO}_{2}$ vs. blank control. (GLM, $\left.{ }^{*} P<0.05, n=12\right)$.

acid $+\mathrm{CO}_{2}$ vs. $\mathrm{dH}_{2} \mathrm{O}$ ) produced significantly greater relative attraction (the proportion of mosquitoes in the arm of the Y-tube containing the test odour stimulus) compared with the $\mathrm{CO}_{2}$ vs. blank control (fig. 2). Relative attraction in response to the $(R)$-1-octen-3-ol enantiomer was significantly greater than

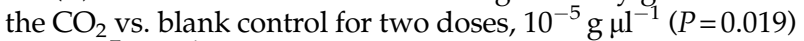

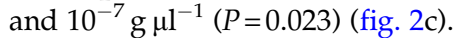

No significant differences were found between the positive control and $\mathrm{CO}_{2}$ vs. blank control for $\mathrm{C} x$. quinquefasciatus mosquitoes (fig. 3). The (R)-1-octen-3-ol enantiomer caused a significantly lower relative attraction than the $\mathrm{CO}_{2}$ vs. blank control at one dose $10^{-3} \mathrm{~g}^{-1}(P<0.001)$ (fig. 3c). A similar result was noted with racemic 1-octen-3-ol and the $(R: S)-1-$ octen-3-ol mixture (84:16) in which the relative attraction to the

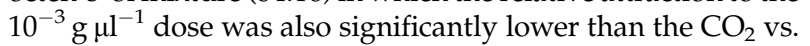
blank control $(P<0.001$ and $P=0.014$, respectively) (fig. 3a,d). No statistically significant differences were found for relative attraction with the (S)-1-octen-3-ol enantiomer (fig. 3b).

\section{Flight activation}

For flight activation (the proportion of mosquitoes that had flown upwind beyond $30 \mathrm{~cm}$ in the stem of the Y-tube) with Ae. aegypti, no significant differences were found for the racemic 1-octen-3-ol or either enantiomer when compared with the $\mathrm{CO}_{2}$ vs. blank control $(P>0.05)$ (table 1). The (R:S)-1-octen-3-ol mixture (84:16) showed significantly less activation at $10^{-8} \mathrm{~g}^{-1}(P=0.030)$ (table 1$)$.

With $C x$. quinquefasciatus, no significant differences were found for racemic 1-octen-3-ol $(P>0.05)$ (table 1). Activation was significantly greater than the $\mathrm{CO}_{2}$ vs. blank control for $(R)$-1-octen-3-ol at $10^{-4} \mathrm{~g} \mu \mathrm{l}^{-1}(P=0.027)$ and $10^{-6} \mathrm{~g} \mathrm{l}^{-1}$ $(P=0.048)$ (table 1$)$. It was noted, generally, that these mosquitoes elicited a lower activation response compared to Ae. aegypti.

No significant differences between the positive control and the $\mathrm{CO}_{2}$ vs. blank control were noted for either mosquito species in relation to flight activation.

\section{Non-sustained flight activity (NSFA)}

In all the experiments with $A$ e. aegypti, the positive control (L-(+)-lactic acid $+\mathrm{CO}_{2}$ vs. $\mathrm{dH}_{2} \mathrm{O}$ ) produced significantly lower NSFA (the proportion of mosquitoes that had flown beyond $30 \mathrm{~cm}$ in the stem of the Y-tube but did not reach the capture chambers at the end of the arms) compared with the $\mathrm{CO}_{2}$ vs. blank control (table 2). The (R:S)-1-octen-3-ol mixture (84:16) elicited a significantly greater NSFA than the $\mathrm{CO}_{2}$ vs. blank control at the $10^{-7}{\mathrm{~g} \mathrm{l}^{-1}}^{-}$dose $(P=0.011)$ (table 2$)$. No significance was found when using $(S)$-1-octen-3-ol $(P>0.05)$ 


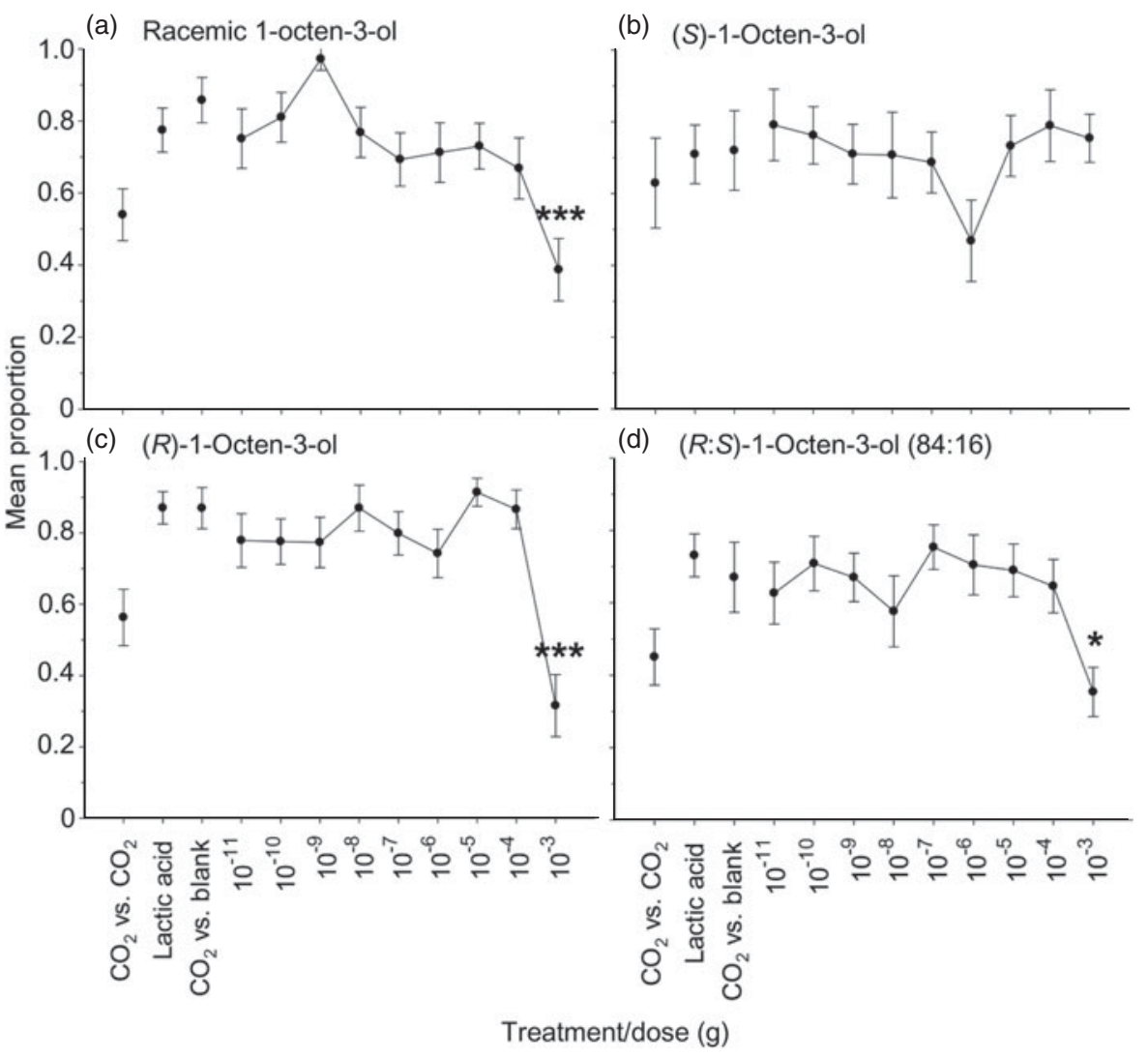

Fig. 3. Relative attraction of Culex quinquefasciatus female mosquitoes in a Y-tube olfactometer to 1-octen-3-ol treatments (plus $\mathrm{CO}_{2}$ ) and controls: $\mathrm{CO}_{2}$ vs. blank, $\mathrm{CO}_{2}$ vs. $\mathrm{CO}_{2}$ and $\mathrm{L}-(+)$-lactic acid $+\mathrm{CO}_{2}$ vs. $\mathrm{dH}_{2} \mathrm{O}$. Relative attraction is the proportion of mosquitoes that flew upwind and were caught in the capture chamber of the treated arm of the Y-tube olfactometer. (a) racemic 1-octen-3-ol, (b) (S)-1-octen-3-ol, (c) (R)-1-octen-3-ol and (d) (R:S)-1-octen-3-ol (84:16) mixture. Asterisks show statistically significant differences in response compared to the $\mathrm{CO}_{2}$ vs. blank control. (GLM, ${ }^{*} P<0.05, * * * P<0.001, n=12$ ).

Table 1. Flight activation of Aedes aegypti and Culex quinquefasciatus female mosquitoes in a Y-tube olfactometer to 1-octen-3-ol treatments (plus $\mathrm{CO}_{2}$ ) and controls: $\mathrm{CO}_{2}$ vs. blank, $\mathrm{CO}_{2}$ vs. $\mathrm{CO}_{2}$ and $\mathrm{L}-(+)$-lactic acid $+\mathrm{CO}_{2}$ vs. $\mathrm{dH}_{2} \mathrm{O}$.

\begin{tabular}{|c|c|c|c|c|c|c|c|c|}
\hline \multirow{2}{*}{$\frac{\text { Activation }}{\text { Treatment per dose }\left(\mathrm{g} \mu \mathrm{l}^{-1}\right)}$} & \multirow[b]{2}{*}{ Racemic } & \multicolumn{3}{|c|}{ Aedes aegypti } & \multirow[b]{2}{*}{ Racemic } & \multicolumn{3}{|c|}{ Culex quinquefasciatus } \\
\hline & & $S$ & $R$ & $R: S$ & & $S$ & $R$ & $R: S$ \\
\hline $\mathrm{CO}$ vs. $\mathrm{CO}_{2}$ & $0.72 \pm 0.05$ & $0.69 \pm 0.05$ & $0.70 \pm 0.04$ & $0.69 \pm 0.05$ & $0.49 \pm 0.05$ & $0.37 \pm 0.05$ & $0.49 \pm 0.05$ & $0.42 \pm 0.05$ \\
\hline $\mathrm{CO}_{2}$ vs. blank & $0.74 \pm 0.05$ & $0.75 \pm 0.04$ & $0.68 \pm 0.04$ & $0.77 \pm 0.05$ & $0.56 \pm 0.05$ & $0.41 \pm 0.05$ & $0.58 \pm 0.05$ & $0.37 \pm 0.05$ \\
\hline $10^{-3}$ & $0.68 \pm 0.05$ & $0.75 \pm 0.04$ & $0.66 \pm 0.04$ & $0.69 \pm 0.05$ & $0.62 \pm 0.05$ & $0.49 \pm 0.05$ & $0.62 \pm 0.05$ & $0.48 \pm 0.05$ \\
\hline $10^{-4}$ & $0.65 \pm 0.05$ & $0.75 \pm 0.05$ & $0.59 \pm 0.04$ & $0.72 \pm 0.05$ & $0.57 \pm 0.05$ & $0.44 \pm 0.05$ & $0.72 \pm 0.04^{*}$ & $0.46 \pm 0.05$ \\
\hline $10^{-7}$ & $0.74 \pm 0.05$ & $0.80 \pm 0.04$ & $0.72 \pm 0.04$ & $0.70 \pm 0.05$ & $0.55 \pm 0.05$ & $0.53 \pm 0.05$ & $0.55 \pm 0.05$ & $0.45 \pm 0.05$ \\
\hline $10^{-8}$ & $0.69 \pm 0.05$ & $0.73 \pm 0.04$ & $0.71 \pm 0.04$ & $0.61 \pm 0.06^{*}$ & $0.55 \pm 0.05$ & $0.38 \pm 0.05$ & $0.59 \pm 0.05$ & $0.36 \pm 0.05$ \\
\hline $10^{-9}$ & $0.73 \pm 0.05$ & $0.83 \pm 0.04$ & $0.76 \pm 0.04$ & $0.64 \pm 0.05$ & $0.61 \pm 0.05$ & $0.51 \pm 0.05$ & $0.65 \pm 0.05$ & $0.49 \pm 0.05$ \\
\hline $10^{-10}$ & $0.69 \pm 0.05$ & $0.83 \pm 0.04$ & $0.73 \pm 0.04$ & $0.63 \pm 0.05$ & $0.63 \pm 0.05$ & $0.52 \pm 0.05$ & $0.60 \pm 0.05$ & $0.37 \pm 0.05$ \\
\hline $10^{-11}$ & $0.74 \pm 0.05$ & $0.84 \pm 0.04$ & $0.76 \pm 0.04$ & $0.64 \pm 0.05$ & $0.49 \pm 0.05$ & $0.36 \pm 0.04$ & $0.55 \pm 0.05$ & $0.33 \pm 0.05$ \\
\hline
\end{tabular}

Flight activation is the proportion of mosquitoes that were activated and flew upwind leaving the release chamber of the Y-tube olfactometer in response to racemic 1-octen-3-ol, (S)-1-octen-3-ol, $(R)-1$-octen-3-ol and (R:S)-1-octen-3-ol (84:16) mixture. Data are presented as mean \pm standard error. Asterisks show statistically significant differences in response compared to the $\mathrm{CO}_{2}$ vs. blank control. (GLM,

* $P<0.05, n=12$ ).

(table 2). The (R)-1-octen-3-ol produced significantly lower

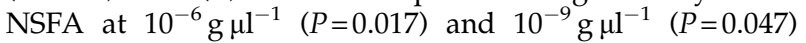
(table 2).
With $C x$. quinquefasciatus mosquitoes, the positive control (L-(+)-lactic acid $+\mathrm{CO}_{2}$ vs. $\mathrm{dH}_{2} \mathrm{O}$ ) produced no significant differences in NSFA compared with the $\mathrm{CO}_{2}$ vs. blank control 
Table 2. Non-sustained flight activity (NSFA) of Aedes aegypti and Culex quiquefasciatus female mosquitoes in a Y-tube olfactometer to 1octen-3-ol treatments (plus $\mathrm{CO}_{2}$ ) and controls: $\mathrm{CO}_{2}$ vs. blank, $\mathrm{CO}_{2}$ vs. $\mathrm{CO}_{2}$ and L-(+)-lactic acid $+\mathrm{CO}_{2}$ vs. $\mathrm{dH} \mathrm{H}_{2} \mathrm{O}$. NSFA is the proportion of mosquitoes that were activated and flew upwind leaving the release chamber, however, failed to reach the capture chambers of the $\mathrm{Y}$-tube olfactometer in response to racemic 1-octen-3-ol, $(S)-1$-octen-3-ol, $(R)-1$-octen-3-ol and (R:S)-1-octen-3-ol (84:16) mixture.

\begin{tabular}{|c|c|c|c|c|c|c|c|c|}
\hline \multirow{2}{*}{$\frac{\text { Non-sustained flight activity }}{\text { Treatment/dose }\left({\left.\mathrm{g} \mu \mathrm{l}^{-1}\right)}\right.}$} & \multirow[b]{2}{*}{ Racemic } & \multicolumn{3}{|c|}{ Aedes aegypti } & \multirow[b]{2}{*}{ Racemic } & \multicolumn{3}{|c|}{ Culex quinquefasciatus } \\
\hline & & $S$ & $R$ & $R: S$ & & $S$ & $R$ & $R: S$ \\
\hline $\mathrm{CO}_{2}$ vs. $\mathrm{CO}_{2}$ & $0.41 \pm 0.05$ & $0.49 \pm 0.05$ & $0.51 \pm 0.05$ & $0.53 \pm 0.05$ & $0.51 \pm 0.06$ & $0.65 \pm 0.06$ & $0.63 \pm 0.06$ & $0.35 \pm 0.06$ \\
\hline $\mathrm{CO}_{2}$ vs. blank & $0.50 \pm 0.05$ & $0.58 \pm 0.04$ & $0.66 \pm 0.05$ & $0.53 \pm 0.05$ & $0.70 \pm 0.05$ & $0.72 \pm 0.05$ & $0.73 \pm 0.05$ & $0.58 \pm 0.07$ \\
\hline $10^{-3}$ & $0.58 \pm 0.05$ & $0.58 \pm 0.04$ & $0.62 \pm 0.05$ & $0.65 \pm 0.05$ & $0.75 \pm 0.04$ & $0.59 \pm 0.05$ & $0.78 \pm 0.04$ & $0.31 \pm 0.05^{* *}$ \\
\hline $10^{-4}$ & $0.57 \pm 0.05$ & $0.58 \pm 0.05$ & $0.53 \pm 0.06$ & $0.55 \pm 0.05$ & $0.72 \pm 0.05$ & $0.75 \pm 0.05$ & $0.74 \pm 0.04$ & $0.39 \pm 0.06^{*}$ \\
\hline $10^{-5}$ & $0.61 \pm 0.05$ & $0.60 \pm 0.04$ & $0.53 \pm 0.06^{*}$ & $0.63 \pm 0.05$ & $0.59 \pm 0.05$ & $0.64 \pm 0.05$ & $0.64 \pm 0.05$ & $0.37 \pm 0.06^{*}$ \\
\hline $10^{-8}$ & $0.49 \pm 0.05$ & $0.59 \pm 0.04$ & $0.54 \pm 0.05$ & $0.64 \pm 0.05$ & $0.68 \pm 0.05$ & $0.75 \pm 0.05$ & $0.80 \pm 0.04$ & $0.52 \pm 0.07$ \\
\hline $10^{-9}$ & $0.52 \pm 0.05$ & $0.63 \pm 0.04$ & $0.51 \pm 0.05^{*}$ & $0.52 \pm 0.05$ & $0.74 \pm 0.04$ & $0.64 \pm 0.05$ & $0.76 \pm 0.04$ & $0.40 \pm 0.06^{*}$ \\
\hline $10^{-10}$ & $0.49 \pm 0.05$ & $0.52 \pm 0.04$ & $0.64 \pm 0.05$ & $0.60 \pm 0.05$ & $0.74 \pm 0.04$ & $0.68 \pm 0.05$ & $0.67 \pm 0.05$ & $0.36 \pm 0.07^{*}$ \\
\hline $10^{-11}$ & $0.46 \pm 0.05$ & $0.61 \pm 0.04$ & $0.70 \pm 0.05$ & $0.52 \pm 0.05$ & $0.71 \pm 0.05$ & $0.71 \pm 0.06$ & $0.74 \pm 0.05$ & $0.38 \pm 0.07^{*}$ \\
\hline
\end{tabular}

Data are presented as mean \pm standard error. Asterisks show statistically significant differences in response compared to the $\mathrm{CO}_{2}$ vs. blank control. (GLM, ${ }^{*} P<0.05,{ }^{* *} P<0.01,{ }^{* * *} P<0.001, n=12$ ).

except for the experiment where the 1-octen-3-ol mixture (84:16 R:S) was tested, in which the response to L-(+)-lactic acid $+\mathrm{CO}_{2}$ was significantly lower $(P=0.002)$ (table 2$)$. No significant differences in NSFA were found using the racemic 1-octen-3-ol or the (R)-1-octen-3-ol enantiomer $(P>0.05)$. The (R:S)-1-octen-3-ol mixture (84:16) elicited a significant decrease in NSFA compared to the $\mathrm{CO}_{2}$ vs. blank

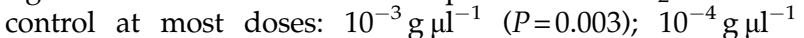

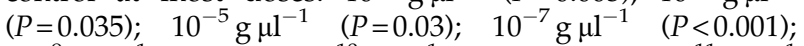

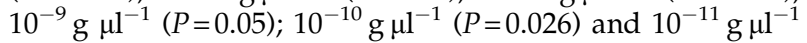
$(P=0.047)$ (table 2).

\section{Electrophysiology}

The B cell, i.e. the olfactory receptor neuron with an intermediate amplitude, of the maxillary palp basiconic sensilla of both species responded to racemic 1-octen-3-ol, as well as the two enantiomers in a dose dependent manner (fig. 4).

In both species, the $\mathrm{B}$ neuron showed a significant difference in selectivity between the enantiomers, with $(R)-1$ octen-3-ol eliciting a greater response, followed by racemic 1-octen-3-ol then (S)-1-octen-3-ol (fig. 4). For Ae. aegypti, (R)-1-octen-3-ol produced significant differences compared to

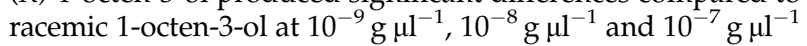
( $P=0.047, P=0.002$ and $P=0.01)$; whereas, significant differ-

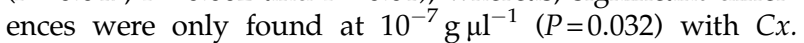
quinquefasciatus (fig. $4 \mathrm{~b}, \mathrm{~d}$ ). In both species, the racemic 1octen-3-ol elicited a significantly greater response compared to

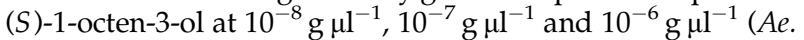
aegypti: $P=0.0005, P=0.0002, P=0.004 ; C x$. quinquefasciatus: $P=0.001, P=0.0003$ and $P=0.004$, respectively). Similarly, comparison of responses to the enantiomers showed that $(R)$ 1-octen-3-ol produced significantly greater responses in both

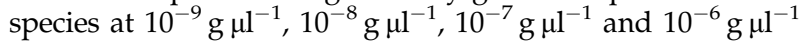
(Ae. aegypti: $P=0.003, P=0.0001, P=0.0001, P=0.0008 ; C x$. quinquefasciatus: $P=0.005, P=0.0003, P=0.0001$ and $P=0.001$, respectively). No significant differences were found for any

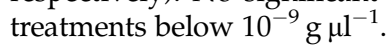

\section{Discussion}

In this study, we investigated the behavioural and electrophysiological responses of Ae. aegypti and $C x$. quinquefasciatus mosquitoes to (R)-1-octen-3-ol, (S)-1-octen-3-ol and to mixtures of the enantiomers in combination with $\mathrm{CO}_{2}$. Previous studies have investigated the role of racemic 1octen-3-ol in mosquito attraction. However, few investigations have examined the effect of the individual enantiomers. In our study, clear electrophysiological and behavioural differences between $A e$. aegypti and Cx. quinquefasciatus were recorded in response to the enantiomers.

Aedes aegypti maxillary palp ORNs detected and discriminated between the $(R)$ and $(S)$ enantiomers at a stimulus concentration of $10^{-9} \mathrm{~g}^{-1}$ and above and discerned (R)-1octen-3-ol from the racemic mixture at concentrations of $10^{-9} \mathrm{~g} \mu \mathrm{l}^{-1}, 10^{-8} \mathrm{~g} \mu \mathrm{l}^{-1}$ and $10^{-7} \mathrm{~g} \mu \mathrm{l}^{-1}$. However, only the (R)-1-octen-3-ol enantiomer elicited a behavioural response in the form of significant increase in relative attraction and a reduction in non-sustained flight activity for Ae. aegypti. The (R:S)-1-octen-3-ol mixture (84:16), based on ratios from cattle breath (Hall et al., 1984), failed significantly to attract either species. However, this could be due to the fact that Ae. aegypti would not normally feed on cattle; and, therefore, it is possible that a different species showing a greater preference for cattle (e.g. certain Anopheles spp.) may elicit significant behavioural results (Takken, 1991). All treatments had little effect in terms of flight activation; however, it was noted that, across all experiments, Ae. aegypti showed a greater average flight activation compared with $C x$. quinquefasciatus. (R)-1Octen-3-ol, (S)-1-octen-3-ol, racemic 1-octen-3-ol and the mixture of the 1-octen-3-ol enantiomers did not elicit a significant relative attraction by $C x$. quinquefasciatus. These results suggest that Ae. aegypti has greater selectivity of the $(R)$ enantiomer at the electrophysiological level and has the most pronounced effect on behaviour, causing activated mosquitoes to sustain their flight and orientate towards the compound. This is the first time electrophysiological selectivity has been shown for a 1-octen-3ol enantiomer in Ae. aegypti in vivo. Bohbot \& Dickens (2009) demonstrated that the in vitro expression of AaOR8 in Xenopus oocytes shows a strong 
(a) Aedes aegypti
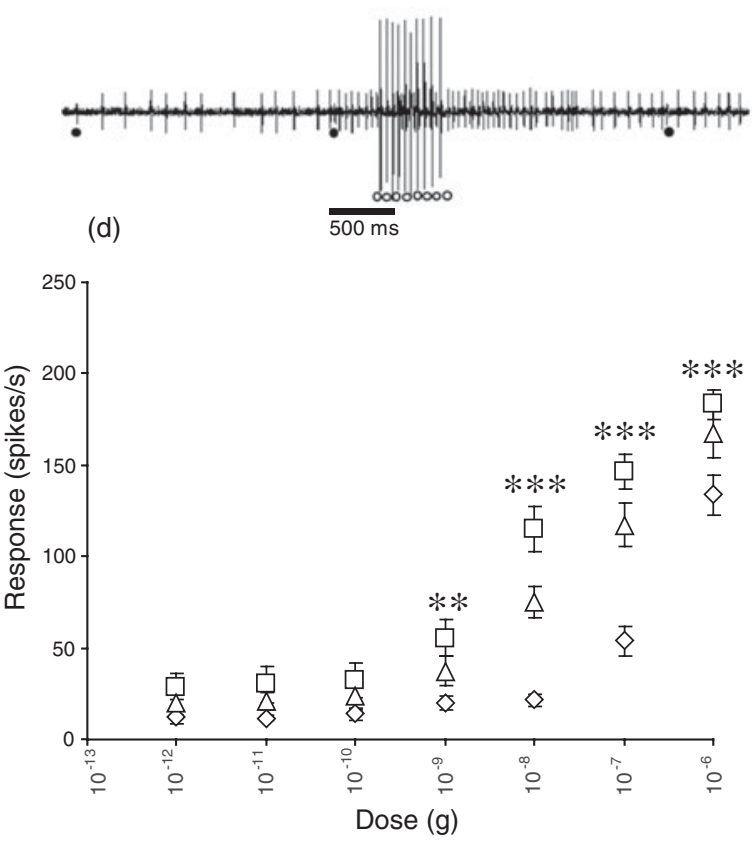

(c) Culex quinquefasciatus

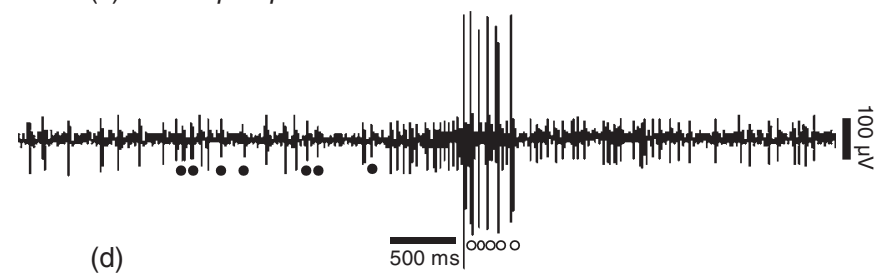

(d)

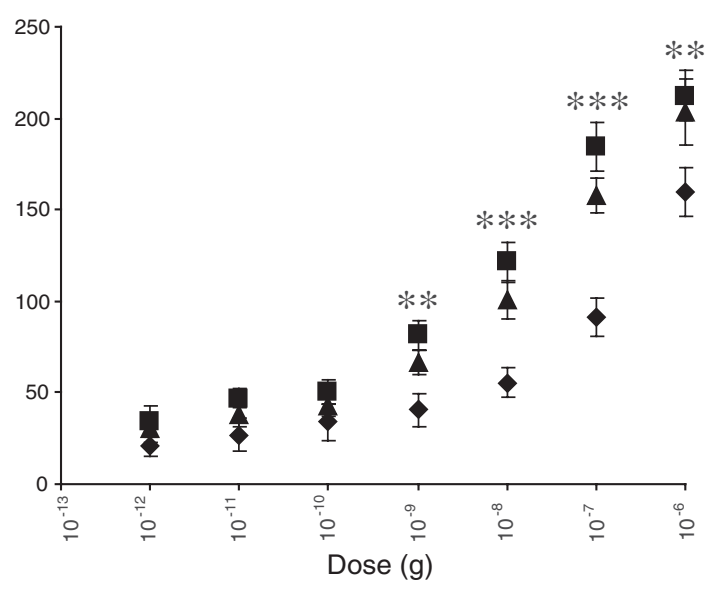

Fig. 4. Dose response relationships for the basiconic sensillum on the maxillary palp in Aedes aegypti and Culex quinquefasciatus female mosquito. 1-Octen-3-ol and its enantiomers elicit dose-dependent responses in the B neuron of basiconic sensilla on the maxillary palp of Ae. aegypti and $C x$. quinquefasciatus female mosquitoes. (a), (c) Response of the B neuron to (R)-1-octen-3-ol and the A neuron (open circle) to ambient $\mathrm{CO}_{2}$ trapped in the stimulus cartridge in Ae. aegypti and $\mathrm{Cx}$. quinquefasciatus, respectively. Closed circles indicate the smallest spiking neuron, the $\mathrm{C}$ neuron. Unmarked intermediate response spikes are from the B neuron. (b), (d) The B neurons in Ae. aegypti and $C x$. quinquefasciatus express significantly higher responses to (R)-1-octen-3-ol as compared to (S)-1-octen-3-ol. (two-way repeated measures ANOVA; $\left.{ }^{*} P<0.05,{ }^{* *} P<0.01,{ }^{* * *} P<0.001\right)$. Significant differences are also found between the response to racemic 1-octen-3-ol and its enantiomers (see results), $n=10$; mean \pm SEM $(\diamond, \mathrm{S}(+) 1$-octen-3-ol Aedes; $\square, \mathrm{R}(-) 1$-octen-3-ol Aedes; $\Delta,(\mathrm{R}+\mathrm{S}) 1$-octen-3-ol Aedes) $(\bullet, \mathrm{S}(+) 1$-octen-3-ol Culex; $\mathbf{\square}, \mathrm{R}(-) 1$-octen-3-ol Culex; $\boldsymbol{\Delta},(\mathrm{R}+\mathrm{S}) 1$-octen-3-ol Culex).

preference towards the $(R)-1$-octen-3-ol enantiomer, which supports our study.

To our knowledge, no other investigation has examined the effect of the 1-octen-3-ol enantiomers on Ae. aegypti behaviour, and few studies have examined the behavioural effect on other mosquitoes species. In the field, Kline et al. (2007) demonstrated that traps baited with $(R)-1$-octen-3-ol, when tested in combination with $\mathrm{CO}_{2}$, caught greater numbers of mosquitoes (Anopheles crucians, Culex salinarius) compared to (S)-1-octen3 -ol, which performed equally or less well than $\mathrm{CO}_{2}$ alone. No significant increase in trap catch of $C x$. quinquefasciatus was recorded. Numerous other studies have reported no significant behavioural effects with racemic 1-octen-3-ol and Culex spp. For example, Culex sitiens and Culex annulirostris have been reported to show either no response or a repellent response to racemic 1-octen-3-ol (Kemme et al., 1993; Van Essen et al., 1994). In Tanzania, a study using unlit CDC traps and racemic 1-octen-3-ol found that the number of $C x$. quinquefasciatus caught did not differ significantly from the unbaited control (Mboera et al., 2000). Similarly, studies of other arthropods have shown a lack of preference for the racemic mixture or enantiomers of 1-octen-3-ol. For example, the bont tick, Amblyomma variegatum, has been shown to display the same orientated movements towards the racemic mixture and (R)-1-octen-3-ol using a servosphere bioassay (McMahon et al., 2001). In a pitfall olfactometer, an equivalent increase in catches of grain beetle, Oryzaephilus mercator, was recorded to the separate 1-octen-3-ol enantiomers and the racemic mixture in comparison to the controls (Pierce et al., 1989).

Culex quinquefasciatus mosquitoes possess ORNs that detect 1-octen-3-ol, and these are located in the basiconic sensilla of the maxillary palps (B neuron). In previous studies, the $B$ cells in maxillary palp sensilla have been shown to have remarkable selectivity, being more sensitive to the (R)-1-octen3-ol enantiomer than the (S) enantiomer (Syed \& Leal, 2007). In An. gambiae it is also the B cell that responds to 1-octen-3-ol (Lu et al., 2007), which suggests that this olfactory receptor neuron (ORN) could be conserved throughout the mosquito lineage. Indeed, in our electrophysiological experiments with Aedes, we found supporting evidence for this and the B cell responded in a similar manner, discriminating between the $(R)$

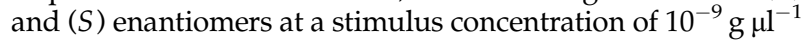
and above and discerning $(R)-1$-octen-3-ol from the racemic mixture at a concentration of $10^{-7} \mathrm{~g}^{-1}$.

Although the (R)-1-octen-3-ol enantiomer caused an increase in activation for $C x$. quinquefasciatus at two doses, the most notable effect was that the (R:S)-1-octen-3-ol mixture (84:16) at seven of the doses tested caused significantly more mosquitoes to sustain their flight and reach the capture chambers (demonstrated by a reduced NSFA), suggesting that it may have a behaviourally excitatory effect. Additionally, at the highest concentration, the few mosquitoes that did reach the capture chambers moved towards the control chamber 
rather than the arm containing the (R)-1-octen-3-ol enantiomer. Interestingly, this reduced relative attraction response was observed for all treatments containing the (R)-1-octen-3-ol enantiomer either on its own or as part of a mixture, but not with the $(S)$-1-octen-3-ol enantiomer. It is unknown whether this effect is true repellency, as the design of the Y-tube olfactometer bioassay does not allow measurement of such behaviour. Similarly, one laboratory study found that $\mathrm{CO}_{2}$ plus racemic 1-octen-3-ol caused a repellent effect in Anopheles quadriannulatus (Pates et al., 2005).

The behavioural differences observed here between Aedes and Culex mosquitoes may be due to feeding preferences as, although both species are known to feed on humans (Takken, 1991; Zinser et al., 2004), Cx. quinquefasciatus can feed opportunistically, and it is preferentially an ornithophilic species (Takken \& Kline, 1989). These results are supported by previous research which shows differences in levels of attraction between mosquito species. For example, one study, using modified Fay-Prince traps to test the effects of $\mathrm{CO}_{2}$ and racemic 1-octen-3-ol on catches of Ae. aegypti, found that it had no effect on increasing trap catches over $\mathrm{CO}_{2}$ alone (Canyon \& Hii, 1997). In contrast, other studies, using racemic 1-octen-3-ol with encephalitis vector surveillance (EVS) traps, showed that 1-octen-3-ol combined with $\mathrm{CO}_{2}$ considerably enhanced catches of Aedes vigilax and Aedes funereus (Kemme et al., 1993; Van Essan et al., 1994). Using CDC light traps in a salt marsh area of North Carolina, USA, it was found that Aedes sollicitans, Aedes taeniorhynchus, Anopheles bradleyi and Culex salinarius were significantly attracted to racemic 1-octen-3-ol in combination with $\mathrm{CO}_{2}$ and light. Additionally, Anopheles atropos showed a highly significant response regardless of the presence of light. Interestingly, when they repeated the experiment in the creek flood plain, none of the 24 species collected were significantly attracted to racemic 1-octen-3-ol in combination with $\mathrm{CO}_{2}$ and light compared to $\mathrm{CO}_{2}$ and light alone (Rueda et al., 2001). More recently, CDC Fay-Prince traps have been used in Maryland, USA, to test the effects of $\mathrm{CO}_{2}$ and racemic 1-octen-3-ol on catches of Aedes albopictus. It was found that, while traps baited with racemic 1-octen-3-ol and $\mathrm{CO}_{2}$ generally caught more Ae. albopictus mosquitoes, this was not significantly different from $\mathrm{CO}_{2}$ alone (Shone et al., 2003). The reason for such varied responses in field trapping could be due to the fact that the racemic form of 1-octen-3-ol does not provide an appropriate cue and therefore results in inconsistent trap catches.

\section{Conclusion}

This study investigated the effects of enantiomers of 1-octen-3-ol using laboratory behaviour bioassays and SSR. In summary, Ae. aegypti mosquitoes differentiate the 1-octen3-ol enantiomers at the electrophysiological level and make oriented movements towards the (R)-1-octen-3-ol enantiomer and do not respond in the same way to either the (S)-1-octen-3-ol enantiomer or to any mixture tested. Flight activation is little affected by the treatments, although a negative behavioural effect was observed in response to the 84:16 (R:S)-1-octen-3-ol mixture. Therefore, the (R)-1octen-3-ol could be a more important kairomone and would be the obvious choice for incorporation into a trap for $A e$. aegypti. Culex mosquitoes also distinguished between the treatments at the electrophysiological level and appeared to be more sensitive to the $(R)$ enantiomer. However, the associated behavioural response was not characteristic of a kairomone. Instead, the mosquitoes made oriented movements away from the $(R)$-1-octen-3-ol enantiomer at high concentrations. Although the mosquitoes were also more activated by this enantiomer than the (S)-1-octen-3-ol enantiomer or any mixture tested, this could be due to an excitatory response and is not necessarily conducive to the development of a suitable attractant for traps for this species. The fact that the SSR response of Culex mosquitoes to the (R)-1-octen-3-ol enantiomer plateaued at the highest concentration suggests that the ORNs became saturated, which may reflect the negative behavioural response observed to this compound at the high concentrations. However, it is worth noting that the behavioural response to the $\mathrm{CO}_{2}$ control was high compared with $A$ e. aegypti even though their overall activation was lower. This could be due to differences in their perception of $\mathrm{CO}_{2}$ (as may be expected due to their feeding habits) and may have masked any fine attractant effects caused by the 1-octen3-ol treatments tested. Further field evaluation of the enantiomers is undoubtedly required. However, the (R)-1octen-3-ol enantiomer could be used for selective trapping for research purposes in areas where Ae. aegypti and $C x$. quinquefasciatus are prevalent. With some further investigation, it may even be possible to use (R)-1-octen-3-ol to interfere with host seeking behaviour of $C x$. quinquefasciatus mosquitoes.

\section{Acknowledgements}

This project was supported by a DTI-LINK grant (J0018 K). We thank Stephen Powers for advice on the statistical analysis and Göran Birgersson for technical help. Rothamsted receives grant-aided support from the Biotechnology and Biological Sciences Research Council (BBSRC) of the United Kingdom. This study was also supported by the Linnaeus-program Insect Chemical Ecology, Ethology and Evolution IC-E ${ }^{3}$.

\section{References}

Becker, N., Zgomba, M., Petric, D. \& Ludwig, M. (1995) Comparison of carbon dioxide, octenol and a host-odour as mosquito attractants in the Upper Rhine Valley, Germany. Medical and Veterinary Entomology 9, 377-380.

Bohbot, J.D. \& Dickens, J.C. (2009) Characterization of an enantioselective odourant receptor in the yellow fever mosquito Aedes aegypti. PloS One 4, e7032.

Burkett, D.A., Lee, W.J., Lee, K.W., Kim, H.C., Lee, H.I., Lee, J.S., Shin, E.H., Wirtz, R.A., Cho, H.W., Claborn, D.M., Coleman, R.E. \& Klein, T.A. (2001) Light, carbon dioxide, and octenol-baited mosquito trap and host seeking activity evaluations for mosquitoes in a malarious area of the Republic of Korea. Journal of the American Mosquito Control Association 17, 196-205.

Bursell, E. (1984) Effects of host odour on the behaviour of tsetse. Insect Science and its Application 5, 345-349.

Canyon, D.V. \& Hii, J.L.K. (1997) Efficacy of carbon dioxide, 1-octen-3-ol, and lactic acid in modified Fay-Prince traps as compared to man-landing catch of Aedes aegypti. Journal of the American Mosquito Control Association 13, 66-70.

Eiras, A.E. \& Jepson, P.C. (1994) Responses of female Aedes aegypti (Diptera: Culicidae) to host odours and convection currents using an olfactometer bioassay. Bulletin of Entomological Research 84, 207-211. 
Gibson, G. \& Torr, S.J. (1999) Visual and olfactory responses of haematophagous Diptera to host stimuli. Medical and Veterinary Entomology 13, 2-23.

Hall, D.R., Beevor, P.S., Cork, A., Nesbitt, B.F. \& Vale, G.A. (1984) 1-Octen-3-ol a potent olfactory stimulant and attractant for tsetse isolated from cattle odours. Insect Science and its Application 5, 335-339.

Hill, S.R., Hansson, B.S. \& Ignell, R. (2009) Characterization of antennal trichoid sensilla from female southern house mosquito, Culex quinquefasciatus Say. Chemical Senses 34(3), 231-252.

Kemme, J.A., Van Essen, P.H.A., Ritchie, S.A. \& Kay, B.H. (1993) Response of mosquitoes to carbon dioxide and 1-octen-3-ol in southeast Queensland, Australia. Journal of the American Mosquito Control Association 9, 431-435.

Khan, A.A., Maibach, H.I., Strauss, W.G. \& Fenley, W.R. (1966) Quantitation of effect of several stimuli on the approach of Aedes aegypti. Journal of Economic Entomology 59, 690-694.

Kline, D.L. \& Lemire, G.F. (1995) Field evaluation of heat as an added attractant to traps baited with carbon dioxide and octenol for Aedes taeniohynchus. Journal of the American Mosquito Control Association 11, 454-456.

Kline, D.L., Allan, S.A., Bernier, U.R. \& Welch, C.H. (2007) Evaluation of the enantiomers of 1-octen-3-ol and 1-octyn-3-ol as attractants for mosquitoes associated with a freshwater swamp in Florida, U.S.A. Medical and Veterinary Entomology 21, 323-331.

Knudsen, J.T., Tollsten, L. \& Bergstrom, G. (1993) Floral scents - A checklist of volatile compounds isolated by headspace techniques. Phytochemistry 33, 253-280.

Logan, J.G., Birkett, M.A., Clark, S.J., Powers, S., Seal, N.J., Wadhams, L.J., Mordue, A.J. \& Pickett, J.A. (2008) Identification of human-derived volatile chemicals that interfere with attraction of Aedes aegypti mosquitoes. Journal of Chemical Ecology 34, 308-322.

Lu, T., Qiu, Y.T., Wang, G., Kwon, J.Y., Rutzler, M., Kwon, H.W., Pitts, R.J., Van Loon, J.J.A., Takken, W., Carson, J.R. \& Zwiebel, L.J. (2007) Odor coding in the maxillay palp of the malaria vector mosquito Anopheles gambiae. Current Biology 17, 1533-1544.

Mboera, L.E.G., Takken, W. \& Sambu, E.Z. (2000) The response of Culex quinquefasciatus (Diptera: Culicidae) to traps baited with carbon dioxide, 1-octen-3-ol, acetone, butyric acid and human foot odour in Tanzania. Bulletin of Entomological Research 90, 155-159.

McMahon, C., Guerin, P.M. \& Syed, Z. (2001) 1-Octen-3-ol isolated from bont ticks attracts Amblyomma variegatum. Journal of Chemical Ecology 27, 471-486.

Miller, R.J., Wing, J., Cope, S., Davey, R.B. \& Kline, D.L. (2005) Comparison of carbon dioxide- and octenol-baited enchepalitis virus surveillance mosquito traps at the Shoalwater Bay Training area, Queensland, Australia. Journal of the American Mosquito Control Association 21, 497-500.
Pates, H.V., Takken, W. \& Curtis, C.F. (2005) Laboratory studies on the olfactory behaviour of Anopheles quadriannulatus. Entomologia Experimentalis et Applicata 114, 153-159.

Pierce, A.M., Pierce, H.D. Jr, Borden, J.H. \& Oehlschlager, A.C. (1989) Production dynamics of cucujolide pheromones and identification of 1-octen-3-ol as a new aggregation pheromone for Oryzaephilus surinamensis and O. mercator (Coleoptera: Cucujidae). Environmental Entomology 18, 747-755.

Rueda, L.M., Harrison, B.A., Brown, J.S., Whitt, P.B., Harrison, R.L. \& Gardner, R.C. (2001) Evaluation of 1octen-3-ol, carbon dioxide, and light as attractants for mosquitoes associated with two distinct habitats in North Carolina. Journal of the American Mosquito Control Association 17, 61-66.

Russell, R.C. (2004) The relative attractiveness of carbon dioxide and octenol in CDC- and EVS-type light traps for sampling the mosquitoes Aedes aegypti (L.), Aedes polynesiensis Marks, and Culex quinquefasciatus Say in Moorea, French Polynesia. Journal of Vector Ecology 29, 309-314.

Shone, S.M., Ferrao, P.N., Lesser, C.R., Glass, G.E. \& Norris, D.E. (2003) Evaluation of carbon dioxide- and 1-octen-3-ol-baited Centers for Disease Control Fay-Prince traps to collect Aedes albopictus. Journal of the American Mosquito Control Association 19, 445-447.

Syed, Z. \& Leal, W.S. (2007) Maxillary palps are broad spectrum odourant detectors in Culex quinquefasciatus. Chemical Senses 32, 727-738.

Takken, W. (1991) The role of olfaction in host-seeking of mosquitoes: a review. Insect Science and its Application 12, 287-295.

Takken, W. \& Kline, D.L. (1989) Carbon dioxide and 1-octen-3-ol as mosquito attractants. Journal of the American Mosquito Control Association 5, 311-316.

Vale, G.A. \& Hall, D.R. (1985a) The role of 1-octen-3-ol, acetone and carbon dioxide in the attraction of tsetse flies, Glossina spp. (Diptera: Glossinidae), to ox odour. Bulletin of Entomological Research 75, 209-217.

Vale, G.A. \& Hall, D.R. (1985b) The use of 1-octen-3-ol, acetone and carbon dioxide to improve baits for tsetse flies, Glossina spp. (Diptera: Glossinidae). Bulletin of Entomological Research 75, 219-231.

Van Essen, P.H.A., Kemme, J.A., Ritchie, S.A. \& Kay, B.H. (1994) Differential responses of Aedes and Culex mosquitoes to octenol or light in combination with carbon dioxide in Queensland, Australia. Medical and Veterinary Entomology 8, 63-67.

Vythilingam, I., Lian, C.G. \& Thim, S. (1992) Evaluation of carbon dioxide and 1-octen-3-ol as mosquito attractants. Southeast Asian Journal of Tropical Medicine and Public Health 23, 328-332.

Zinser, M., Ramberg, F. \& Willott, E. (2004) Culex quinquefasciatus (Diptera: Culicidae) as a potential West Nile virus vector in Tuscon, Arizona: blood meal analysis indicates feeding on both humans and birds. Journal of Insect Science 4, 20. 